\title{
Identification of Materials Mechanical Properties from Full-Field Measurements: Latest Advances in the Virtual Fields Method
}

\author{
F. Pierron \\ LMPF, Arts et Metiers ParisTech, Rue St-Dominique, BP 508 \\ 51000 Châlons en Champagne Cedex, France \\ fabrice.pierron@chalons.ensam.fr
}

\begin{abstract}
Keywords: Full-field measurements, virtual fields method, inverse identification, material mechanical properties
\end{abstract}

\begin{abstract}
This paper presents a short overview of the state of the art and future challenges of the use of full-field measurements and inverse procedures to identify the constitutive mechanical parameters of a wide range of materials. It concentrates on the so-called Virtual Fields Method (VFM) which is a tool fully dedicated to the processing of full-field measurements. Some of the future challenges are briefly covered here, namely the design of test configurations and the application to damage assessment, high strain rate testing and biomaterials. Some examples are given and the main scientific issues briefly discussed.
\end{abstract}

\section{Introduction}

The fast development of full-field optical methods (digital image correlation, grid methods, speckle Interferometry, etc.) has dramatically changed the prospects of the mechanical testing of materials and structures. Whereas local strain measurements over a limited number of points with strain gauges or LVDTs restricted the testing configurations to simple shapes and load cases (uniaxial tension or compression, three-point bending etc.), kinematic measurements over tens to hundreds of thousands of points now enable much more complex situations to be addressed. In particular, one can perform the simultaneous determination of several stiffness parameters for anisotropic materials, complex elasto-plastic laws and heterogeneous materials (including welds and damaged composites). Recently, this area of research has dramatically expanded and significant progress has been made towards the production of automated tools to help testing engineers and scientists. The objective of the present paper is to provide an overview of the state of the art and the future challenges awaiting the Virtual Fields Method (VFM), which is an inverse identification procedure fully dedicated to full-field measurements.

\section{State of the art}

The main objective of the VFM is to process full-field kinematic measurements to identify parameters driving the constitutive behaviour of materials. The present paper will not review the method in detail, this can be found in [1,2]. Only the main features will be highlighted. It must also be noted that the VFM is an alternative to a more general approach often referred to as Finite Element Model Updating (FEMU). Again, more details can be found in [2,3]. In short, the main difference between the two is that FEMU recalculates the mechanical fields iteratively from external load and geometry whereas the VFM processes the measured fields directly to assess equilibrium, hence leading to much shorter computation times.

Homogeneous linear elasticity. Historically, this was the first application of the VFM. The motivation was aimed at the simultaneous identification of all the anisotropic in-plane or bending stiffness components of composite plates [4-5]. Basically, the idea is to write the principle of virtual work, as in Eq. 1 below (in the static case of static and without body forces): 


$$
-\int_{\mathrm{V}} \sigma_{\mathrm{ij}} \varepsilon_{\mathrm{ij}}^{*} \mathrm{dV}+\int_{\partial \mathrm{V}} \mathrm{T}_{\mathrm{i}} \mathrm{u}_{\mathrm{i}}^{*} \mathrm{~d} \mathrm{~S}=0
$$

where $\sigma$ is the stress tensor, $\mathrm{T}$ the distribution of surface loads, $\mathrm{u}^{*}$ the virtual displacement field, $\varepsilon^{*}$ the associated virtual strain field, $\mathrm{V}$ the volume of the solid and $\partial \mathrm{V}$ its boundary. Assuming a linear elastic behaviour with $\sigma_{\mathrm{ij}}=\mathrm{C}_{\mathrm{ijk}} \varepsilon_{\mathrm{kl}}$, one can substitute the stress tensor into Eq. 1 and provided that the material is homogeneous, the $\mathrm{C}$ components can be taken out of the integrals to give:

$$
-\mathrm{C}_{\mathrm{ijk} 1} \int_{\mathrm{V}} \varepsilon_{\mathrm{kl}} \varepsilon_{\mathrm{ij}}^{*} \mathrm{dV}+\int_{\partial \mathrm{V}} \mathrm{T}_{\mathrm{i}} \mathrm{u}_{\mathrm{i}}^{*} \mathrm{dS}=0
$$

Therefore, for each choice of kinematically admissible virtual field, a linear equation can be established linking some of the elastic unknowns to integrals of the actual strains that can be evaluated if full-field measurements are available. The methodology was successfully applied to plates in bending [4] and to in-plane properties [5] using virtual fields defined manually, but it soon became clear that the choice of the virtual fields had a large impact on the quality of the identification. To overcome this, a procedure to define optimized virtual fields was devised [6] and excellent experimental results were obtained with this approach [7]. A formal link was also established between the VFM and FEMU techniques for the case of linear elasticity [8] and it can now be concluded that from a theoretical point of view, the problem has been solved for homogeneous linear elasticity.

Homogeneous elasto-plasticity. More recently, the VFM was extended to elasto-plastic behaviour [9]. In this case, the procedure described previously does not apply because the stress is not explicitly a function of the total measured strain. One has therefore to resort to an incremental procedure. First, a constitutive behaviour is assumed and initial parameters are selected. At each step, using the total measured strains and the elasto-plastic parameters, the stresses can be calculated and used to build up a cost function as:

$$
\mathrm{f}\left(\varepsilon^{\mathrm{t}}, \mathrm{E}, \mathrm{v}, \mathrm{X}_{1} \ldots \mathrm{X}_{\mathrm{p}}\right)=\sum_{\mathrm{k}=1}^{\mathrm{n}}\left(-\int_{\mathrm{V}} \sigma_{\mathrm{ij}}^{\mathrm{k}} \varepsilon_{\mathrm{ij}}^{*} \mathrm{dV}+\int_{\partial \mathrm{V}} \mathrm{T}_{\mathrm{i}}^{\mathrm{k}} \mathrm{u}_{\mathrm{i}}^{*} \mathrm{dS}\right)^{2}
$$

where $\varepsilon^{t}$ is the measured total strain tensor, $\mathrm{E}$ and $v$ the elastic constants, $\mathrm{X}_{1}$ to $\mathrm{X}_{\mathrm{p}}$ the $\mathrm{p}$ elastoplastic parameters to be identified and $\mathrm{k}$ the number associated with the $\mathrm{k}^{\text {th }}$ load step. The minimization leads to the unknown parameters. It is important to note that although this procedure is now iterative, it is still much faster than FEMU since no forward problem has to be solved. Typically, it runs in a few minutes. Successful experimental validation was obtained [10,11] and in practice, the choice of a single simple virtual field gives good results. Further optimization of the virtual fields for this case is underway but it does not seem to be as critical as for the elastic properties.

The Camfit software. One of the main problems facing the uptake of this type of procedure (heterogeneous test, full-field measurement and inverse identification) is the fact that testing engineers and scientists do not necessarily wish to program the inverse routines themselves. This is rather complex and requires a good understanding of the topic. Therefore, it is absolutely necessary to be able to provide an integrated software tool that could process the full-field measurements automatically and provide the constitutive parameters. This is the philosophy of the Camfit software. Based on the above, it can presently tackle anisotropic in-plane stiffnesses and simple elasto-plastic laws (linear or non-linear isotropic hardening). A GUI has been designed (see Fig. 1) and further details of the software can be found in $[12,13]$. 


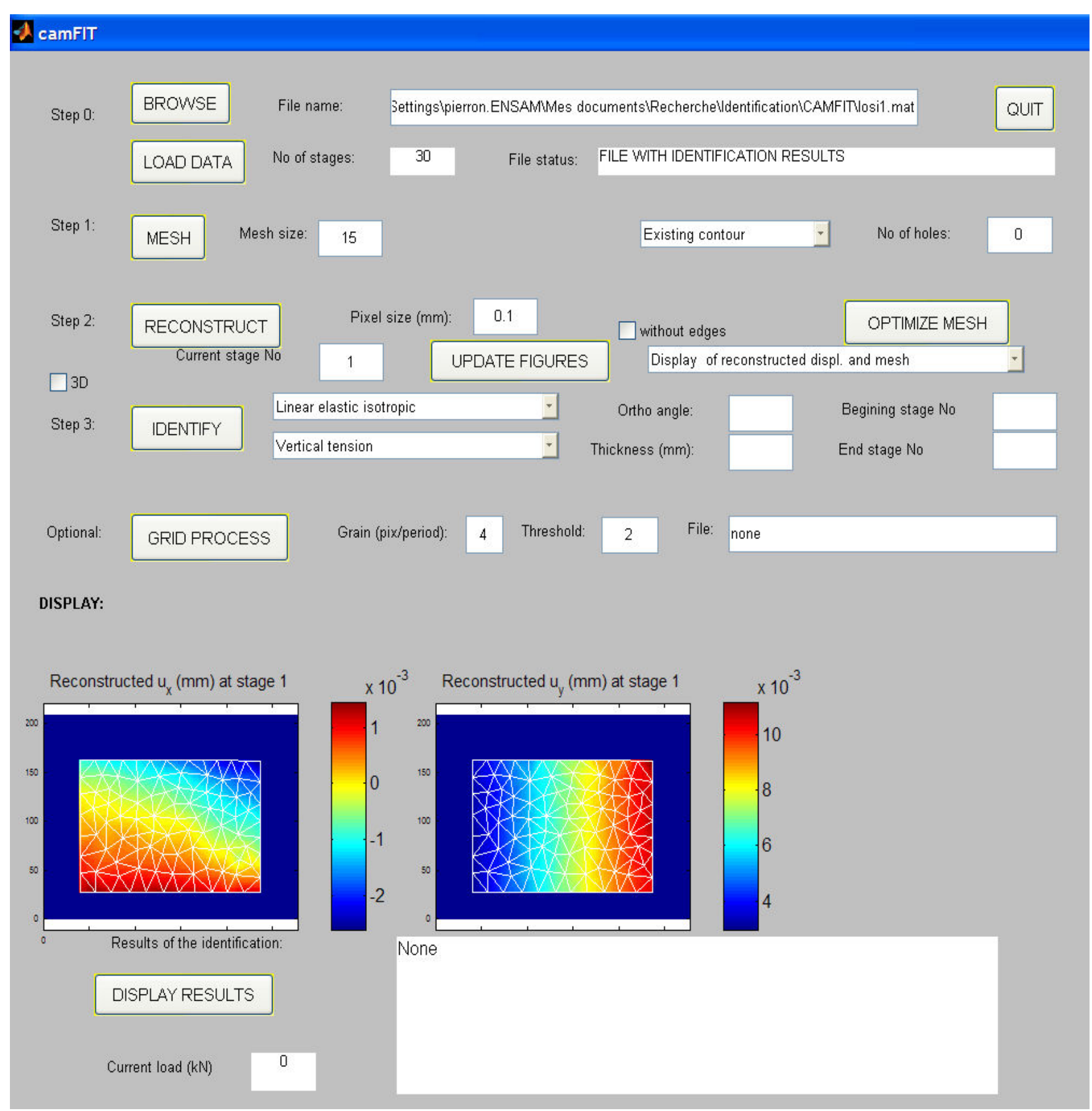

Fig. 1. CamFit GUI.

\section{Recent developments and future challenges}

There are many challenges to be addressed in future years and it is beyond the scope of the present article to attempt a complete list of these. Some of the key problems of particular significance are detailed below.

Test design and optimization. Since the restrictions on shape and loading conditions are relaxed with the present procedure, the choice of test configuration must be made using specific criteria. In the case of orthotropic elasticity for instance, one criterion would be that all stiffness components are identified with the same level of confidence. In other words, the sensitivity of the actual mechanical fields to the different components of the stiffness tensor should be balanced. A few attempts at test design can be found in the literature, mainly for the bending of thin plates, either using FEMU [14] or the VFM [15]. For in-plane properties, an attempt at optimizing the unnotched 
Iosipescu test was made by adjusting the free length $\mathrm{L}$ and the fibre angle theta of the specimen (see Fig. 2).
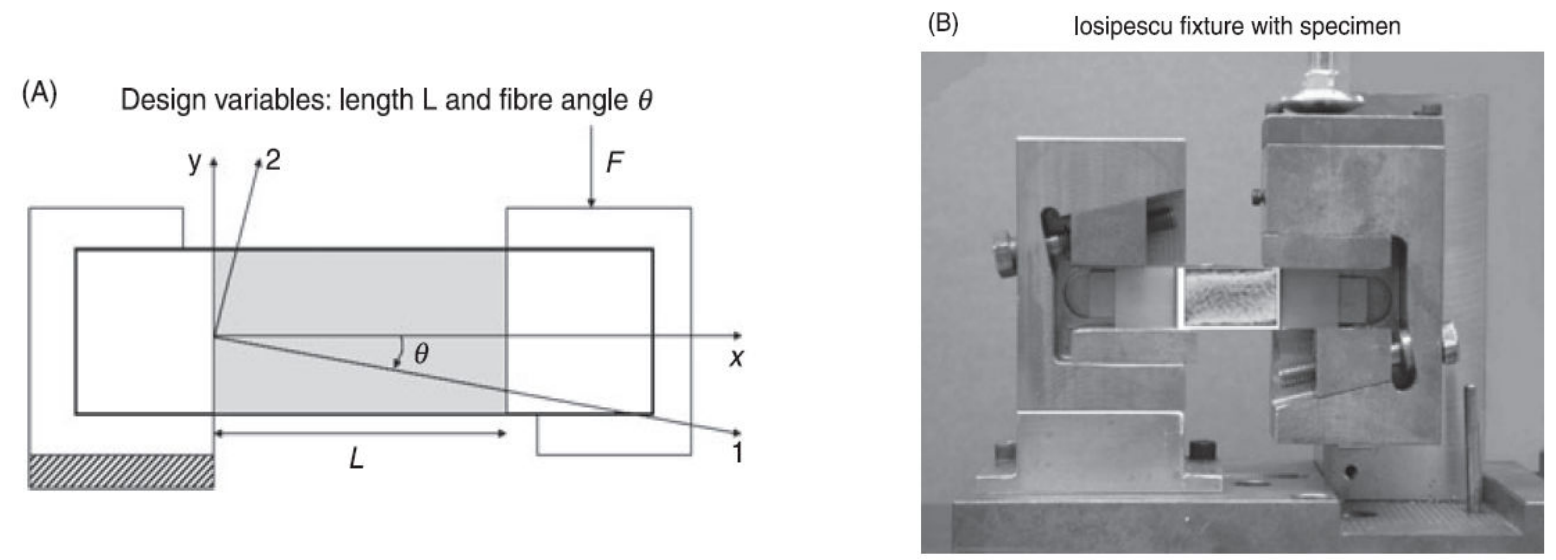

Fig. 2. Unnotched Iosipescu test with design variables.

It was found that angles around $30^{\circ}$ were favourable for a unidirectional glass-epoxy [16]. Nevertheless, these attempts are few and still rather crude. Ideally, the optimization chain should also take into account the imaging system, with its noise and spatial sampling. In particular, a shape optimization will be strongly dependant on the aspect ratio of the CCD sensor. To the best knowledge of the present author, this has still to be addressed and is certainly one of the major issues in the coming years.

Damage in materials. One of the largest potential areas of application of the methodology proposed here is the study of the damage of materials. Two main types of problems can be distinguished: following the degradation of the material during the loading $[17,18]$ or identifying a damage already present, in the spirit of NDT techniques [19]. Very few applications exist in the literature and this remains a significant challenge for the future, with many potential applications. For instance, Fig. 3 shows the bending stiffness reduction map obtained experimentally on a carbonepoxy unidirectional plate tested in bending [19]. Full-field slopes were measured by a deflectometry technique and the curvature used to obtain the coefficients of a polynomial driving the stiffness reduction (considering that all stiffness components were reduced in the same way in the damaged area, that is, the damage is isotropic). The damage was simulated by removing two plies from the back surface of the plate, resulting in a nominal stiffness reduction of about $30 \%$. The results are satisfactory but a lot of work remains to tackle anisotropic damage as well as mid-plane delaminations.

High strain rate testing. Presently, the testing of materials at high strain rates relies on Hopkinson bars and related methods which are macroscopic and cannot follow the localization in the materials. A major challenge for establishing more representative constitutive equations would be to measure the full strain fields using high speed cameras. Presently, the technology is available to capture 5000 images per second for a full $1 \mathrm{k} \mathrm{x} 1 \mathrm{k}$ pixels resolution, but this does not reach strain rates much above $10 \mathrm{~s}^{-1}$ typically. The alternative is to use ultra-high speed cameras based on multiplexed

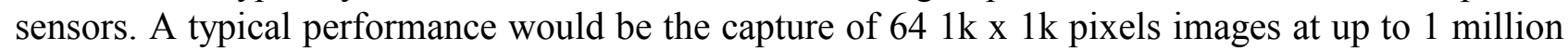
frames per second. However, this is still very difficult because these cameras are not designed as measuring devices. Many artefacts exist that must be corrected for, and there are also difficult issues with consistent lighting. An interesting discussion on this topic can be found in [20]. Some applications have been attempted recently for elasto-plastic materials $[21,22]$ but the subject is still in its infancy, particularly for strain rates above $100 \mathrm{~s}^{-1}$, using ultra-high speed cameras. 
Extraordinary progress in the mechanics of materials in this area is to be expected in the coming years.

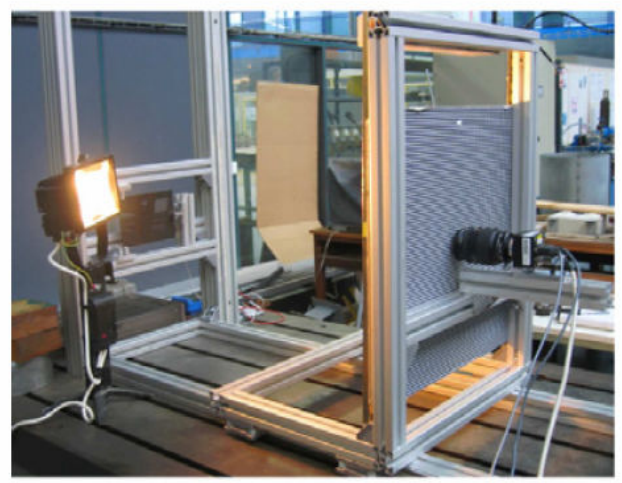

Deflectometry set-up

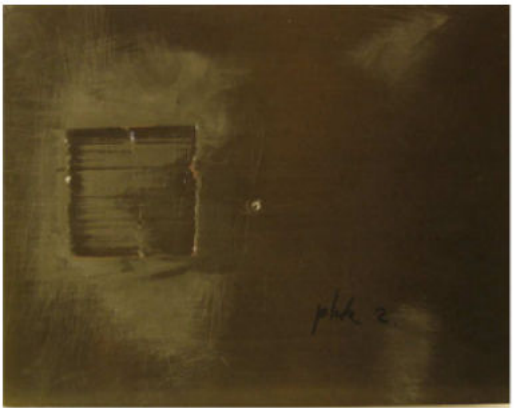

Picture of the other side of inspection

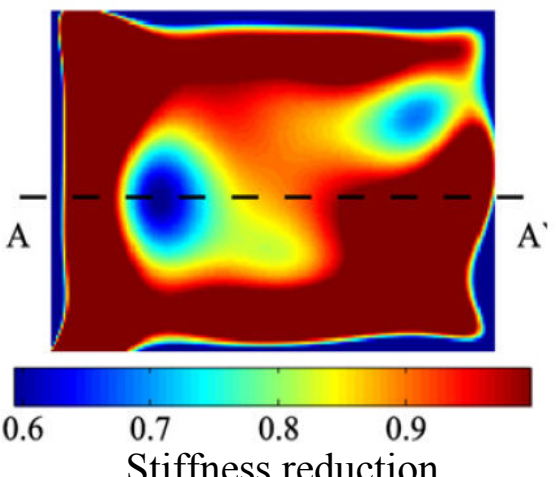

Fig. 3. Deflectometry set-up, damaged plate and identified stiffness reduction map.

Biomaterials. Finally, it is worth mentioning that the progress of medical imaging now enables us to obtain in-situ images of the deformation of biological tissues using ultrasonic (US) or magnetic resonance imaging (MRI) systems. Determination of the mechanical behaviour of biological tissues is a key issue in the early detection of cancerous tumours (stiff tumours embedded in softer tissues) or in the production of data to establish models of the human body for crash simulation, for instance. The determination of stiffness from such measurements is known as "elastography" in the medical community [23,24]. These tissues also exhibit highly non linear behaviour (hyperviscoelastic for muscle tissues, for instance) and there is still much work to do both on the measurements themselves (moving from images to high quality deformation measurements) and on the identification procedures. For the latter, the experience of the mechanics of materials community would be very helpful. Joint projects between medical and engineering groups should be established in the near future to help solve these very important and challenging problems. A first application of the VFM in this area was published recently [25] and a project is presently underway with Loughborough University on the measurement of the stiffness variation of cornea in the throughthickness direction using optical coherence tomography-based techniques [26].

\section{Conclusion}

This paper has presented a short overview of the state of the art and of some challenges in the use of full-field measurements and inverse procedures to extract mechanical constitutive parameters of a large range of materials. More specifically, the paper has focused on a technique fully dedicated to the processing of full-field measurements: the Virtual Fields Method (VFM). 
One of the main conclusions is that the VFM is becoming ready for certain applications, such as elastic stiffness determination (either isotropic or anisotropic) from complex test configurations. A user-oriented software is being developed to help potential users employ the method without having to program the whole routines themselves. Nevertheless, a very large field of research is now open on many issues where engineers struggle, like highly non-linear materials, assessment of damage, heterogeneous materials, welds etc. Only few research groups worldwide are actively engaged in this topic and more efforts are required to speed up progress and establish future standard test procedures making full use of full-field measurements. It is expected that the coming ten years will see a fast growth of this scientific area that will eventually deeply change the everyday life of mechanical testing engineers and mechanics of materials scientists.

\section{Acknowledgements}

The author would like to thank Dr Stéphane Avril and Professor Michel Grédiac for the very fruitful collaboration that, over the years, has resulted in the present state of the art of the Virtual Fields Method.

\section{References}

[1] M. Grédiac, F. Pierron, S. Avril, E. Toussaint: Strain Vol. 42 (2006), p. 233-253.

[2] F. Pierron, S. Avril: EOLSS encyclopedia, http://www.eolss.net (2008).

[3] S. Avril, M. Bonnet, A.-S. Bretelle, M. Grédiac, F. Hild, P. Ienny, F. Latourte, D. Lemosse, S. Pagano, E. Pagnacco, F. Pierron, Experimental Mechanics (2008), accepted.

[4] M. Grédiac, A. Vautrin: Journal of Applied Mechanics Vol. 57 (1990), p. 964-968.

[5] M. Grédiac, F. Pierron, Y. Surrel: Experimental Mechanics Vol. 39 (1999), p. 142-149.

[6] S. Avril, M. Grédiac, F. Pierron: Computational Mechanics Vol. 34 (2004), p. 439-452.

[7] R. Moulart, S. Avril, F. Pierron: Composites Part A Vol. 37 (2006), p. 326-336.

[8] S. Avril, F. Pierron: International Journal of Solids and Structures Vol. 44 (2007), p. 49785002 .

[9] M. Grédiac, F. Pierron: International Journal of Plasticity Vol. 22 (2006), p. 602-627.

[10] Y. Pannier, S. Avril, R. Rotinat, F. Pierron: Experimental Mechanics Vol. 46 (2006), p. 735755.

[11] S. Avril, F. Pierron, Y. Pannier, R. Rotinat: Experimental Mechanics (2008), in press.

[12] V.T. Tran, S. Avril, F. Pierron: in Applied Mechanics and Materials vol. 7-8, edited by J. Quinta da Fonseca, TransTech publications, 2007, p. 57-62.

[13] Information on http://www.camfit.fr

[14]L. Le Magorou, F. Bos, F. Rouger: Composites Science and Technology, Vol. 62 (2002), p. 591-596.

[15] K. Syed-Muhammad, E. Toussaint, M. Grédiac, S. Avril, J.H. Kim, Composite Structures, Vol. 85, (2008), p. 70-82.

[16]F. Pierron, G. Vert, R. Burguete, S. Avril, R. Rotinat, M. Wisnom: Strain, Vol. 43 (2007), p. 250-259.

[17]H. Chalal, S. Avril, F. Pierron, F. Meraghni: Composites Part A, Vol. 37 (2006), p. 315-325. 
[18]D. Claire, F. Hild, S. Roux: International Journal of Damage Mechanics, Vol. 16 (2007), p. 179-197.

[19] J.-H. Kim, F. Pierron, M. Wisnom, K. Syed-Muhamad: Composites Part A, Vol. 38 (2007), p. 2065-2075.

[20] V. Tiwari, M.A. Sutton, S.R. McNeill: Experimental Mechanics, Vol. 47 (2007), p. 561-579.

[21] J. Kajberg, B. Wikman: International Journal of Solids and Structures, Vol. 44 (2007), p. 145164.

[22] S. Avril, F. Pierron, J. Yan, M.A. Sutton: Mechanics of Materials (2008), in press.

[23] A.R. Skovoroda, S.Y. Emelianov, M. O’Donnell: IEEE Trans. Ultrason. Ferroelectr. Freq.

Control, Vol. 42 (1995), p. 747-765.

[24]E. Park, A.M. Maniatty: Phys. Med. Biol. Vol. 51 (2006), p. 3697-3721.

[25] S. Avril, J.M. Huntley, F. Pierron, D.D. Steele: Experimental Mechanics (2008), in press.

[26] P.D. Ruiz, J.M. Huntley, A. Maranon: Proceedings of the Royal Society A: Mathematical, Physical and Engineering Sciences, Vol. 462 (2006), p. 2481-2502. 